\title{
First measurements of top quark properties with Run-2 data in CMS
}

\author{
Kai-Feng Chen* \\ National Taiwan University \\ E-mail: kf jack@phys.ntu.edu.tw \\ on behalf of the CMS Collaboration
}

\begin{abstract}
Measurements of top quark properties using data collected by the CMS experiment at $13 \mathrm{TeV}$ are presented. The top quark mass is measured in the lepton+jets channel is consistent with the CMS measurements of Run-1. The top quark mass is also studied as a function of the event kinematical properties. For the first time at the LHC, the width of the top quark is directly probed during Run-2, in what constitutes the most precise direct bound of the top quark width performed to date. Finally, searches for flavor-changing neutral currents involving top quarks are also discussed including tZq, and tHq couplings, in top quark pair and single top production.
\end{abstract}

XXVI International Workshop on Deep-Inelastic Scattering and Related Subjects (DIS2018)

16-20 April 2018

Kobe, Japan

${ }^{*}$ Speaker. 
In the standard model (SM), the top quark is a particular object, which is the heaviest known point-like particle to date. Due to the heaviness of top, it strongly couples with the Higgs boson in the SM. Hence it does play a significant role also in the Higgs physics, which is the core of the electroweak symmetry breaking. On the other hand, the lifetime of the top quark is found to be very short and decays before its hadronization. Hence it is the only free quark, without any dilution from the hadronic effect of a bound state, and allows experimentalists to access to the top quark properties directly. Measurements of top quark properties with high precisions are good tests of the SM. Any deviations from the prediction would be a clean hint of new physics beyond the SM.

The large hadron collider (LHC) is an ideal place to probe top quark properties: the production cross section is large due to the high center of mass energy, while the LHC has delivered a sizable amount of proton collisions to the experiments already. Many measurements have been performed with the LHC Run-1 data already. The Run-2 data, which is collected at an even higher collision energy, would significantly improve the statistics power further. In this article several new measurements performed with Run-2 data recorded by the CMS detector [1] are presented.

\section{Measurement of top quark mass}

Top quark mass is an essential parameter of the SM. It plays an important role on the stability of the electroweak vacuum of the universe. The measurements with previous Run-1 data are excellent and the results are already dominant by the systematic uncertainties. Understanding of the theoretical interpretation of the measurements is also one of the key issues to pursuit. A direct measurement of top mass based on the classical Ideogram method is performed with $13 \mathrm{TeV}$ data [2]. In the analysis, the top mass is evaluated simultaneously with the jet energy scale factor (JSF) in joint likelihood fits. The fits are performed on the following observables: the fitted top quark mass $m_{\mathrm{t}}^{\text {fit }}$, the reconstructed $\mathrm{W}$ boson mass $m_{W}^{\text {reco }}$, and the in-situ estimator of JSF. The treatment of model uncertainties has been updated. A "hybrid" analysis, which also includes the prior on JSF from jet energy correction measurements, has been carried out and the following results are derived:

$$
\begin{aligned}
m_{\mathrm{t}} & =172.25 \pm 0.08(\text { stat }+\mathrm{JSF}) \pm 0.62(\text { syst }) \mathrm{GeV}, \\
J S F & =0.996 \pm 0.001(\text { stat }) \pm 0.008(\text { syst }) .
\end{aligned}
$$

Figure 1 shows the distributions of the key observables $m_{W}^{\text {reco }}$ and $m_{\mathrm{t}}^{\text {fit }}$ after event selection, and comparing the distributions from data and simulations.

As one of the main systematic uncertainties of the mass measurement is estimated by a comparison of different models. It would be beneficial to perform differential measurements of top quark mass as functions of kinematic properties of $t \bar{t}$ system. Such a study can be a good validation of models, probing potential effects from color reconnection, initial state radiation or final state radiation, and the kinematics of the jets coming from the top decays. As presented in Fig. 2, the measured top masses as functions of the invariant mass of $t \bar{t}$ system, as well as the $\Delta R$ between the light-quark jets show no indications of a kinematical bias in the measurements, found in the comparison between data and simulated events. 

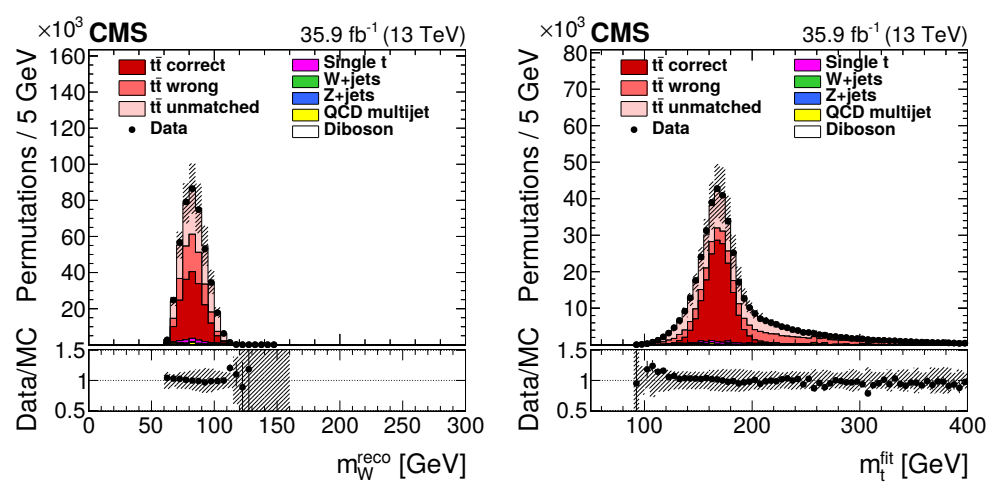

Figure 1: Reconstructed W boson masses $m_{W}^{\text {reco }}$ (left) and fitted top quark masses $m_{\mathrm{t}}^{\text {fit }}$ (right). The distributions of the simulated events are normalized according to the integrated luminosity. See Ref. [2] for detailed information.
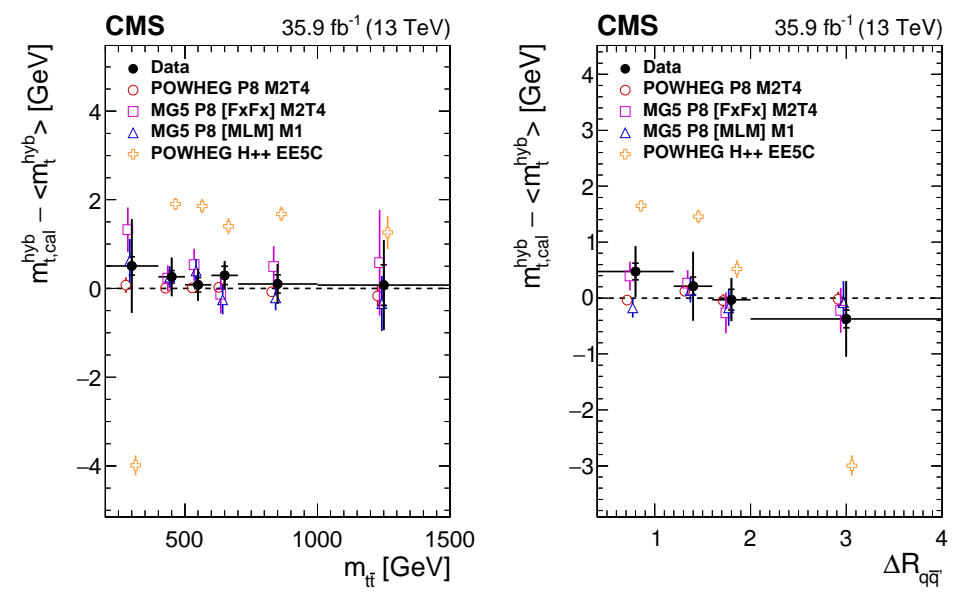

Figure 2: Measurements of top quark mass as a function of the invariant mass of the t⿱t system (left) and the $\Delta R$ between the light-quark jets (right). Data is compared with the distributions produced by different generator models. See Ref. [2] for detailed information.

\section{Bounding top quark width}

The width of the top quark, which is predicted to be around $1.35 \mathrm{GeV}$ in the $\mathrm{SM}$, is another important fundamental property. It is much less discussed and less accurately determined comparing to the mass value itself. There are two ways to determine the width of top quark: an indirect method by combining the branching fraction measurement and single top t-channel cross section, and a direct method by reconstructing top mass line shape and comparing to Monte Carlo simulated predictions. Based on the CMS measurements, the top quark width derived by the indirect method is given by $\Gamma_{\mathrm{t}}=1.36 \pm 0.02_{-0.11}^{+0.14} \mathrm{GeV}$.

A direct measurement of the top quark width has been performed with a high purity dilepton and two jets events [3]. The study is based on the observables which partially reconstruct the 
top quark kinematics. Several different observables based on the top pair kinematics have been tested. The optimal choice found is the inclusive combinations of $b$-jet and lepton invariant masses, where there are up to four combinations of lepton and b-jet per event. As shown in Fig. 3, the ratios between simulated events generated with different mass and width hypotheses show different behavior near the kinematical endpoint.
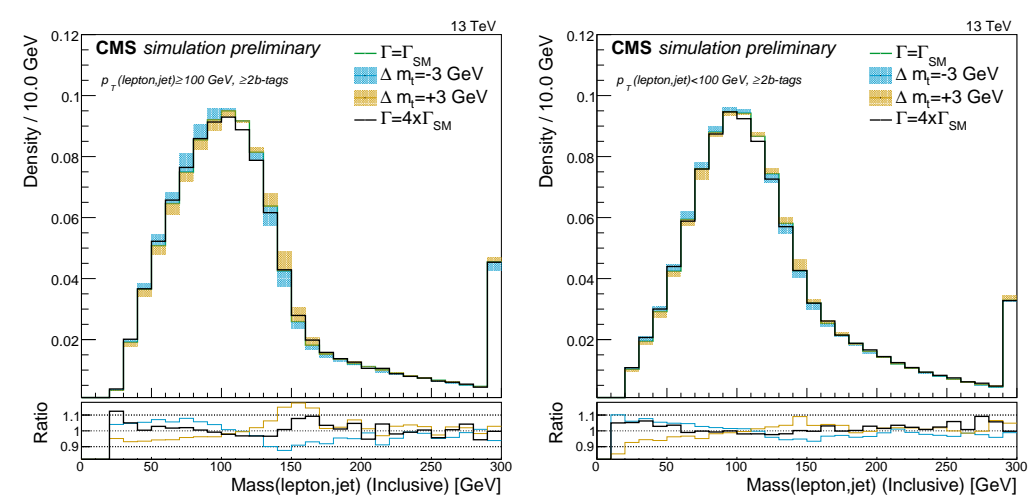

Figure 3: Distribution of the $M_{\ell b}$ variable for the simulated events with different the top quark masses in the generation as well as variation on the width, up to a factor of 4 with respect to the SM value. The unboosted events (left) and boosted events (right) are both shown. See Ref. [3] for detailed information.

Boundaries on top quark width are extracted using a binary hypothesis test. Test statistic is defined by the likelihood ratios between the alternative and the SM hypotheses, and the separation of hypothesis is measured via the $C L_{S}$ criterion. Figure 4 shows the evolution of the $C L_{S}$ as a function of the top quark width. The observed bounds at the $95 \%$ confidence level are

$$
0.6 \mathrm{GeV} \leq \Gamma_{t} \leq 2.5 \mathrm{GeV}
$$

which is the most precise direct bound on top quark width to date.

\section{Search for Top FCNC decays with three-lepton final state}

Flavour-changing neutral current (FCNC) transitions are forbidden at tree level by the GIM mechanism. They are still possible in the SM in the higher orders via loop induced processes, but are highly suppressed in the top decays. Some extensions of the SM could enlarge the FCNC decay rates significantly, by including new physics particles in the loop process, including SUSY and technic color. Furthermore, top FCNC decays may contain distinctive event signatures, including a $\mathrm{Z}$ boson or an isolated photon. The detection of these events is not difficult for the experiments. The decay involves the Higgs boson (FCNC with Higgs) bring in a more richer signatures and physics.

A search focusing on the evidence of a FCNC tZq vertex has been performed by CMS experiment [4], where the quark q can be either an up or charm. The tZq vertex leads to a single top quark production though $\mathrm{q} \rightarrow \mathrm{tZ}$, or a top quark pair production with one of the top quarks decaying with $\mathrm{t} \rightarrow \mathrm{qZ}$ subprocess. When the $\mathrm{W}$ and $\mathrm{Z}$ boson decay leptonically, the event contains a clean three 


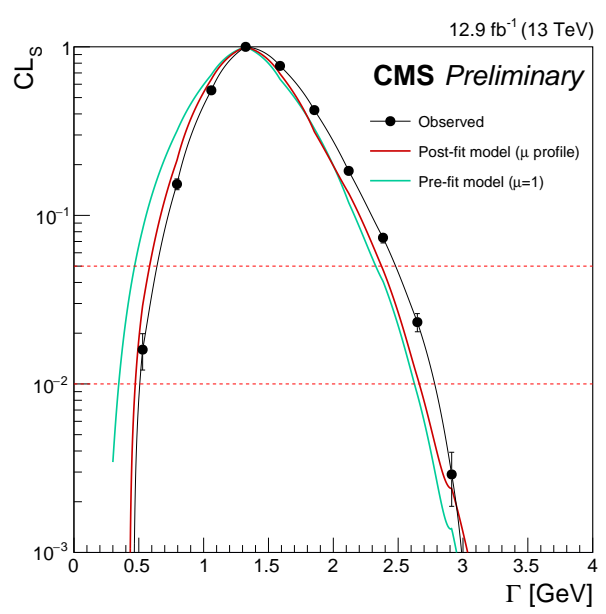

Figure 4: The calculated $C L_{S}$ values as a function of the top quark width; the observed boundaries at the $95 \%(99 \%)$ confidence level are given by the intersection of the fitted curve to the horizontal lines at $C L_{S}=$ 0.05 (0.01). See Ref. [3] for detailed information.

lepton final state. The analysis also takes at least one, up to three jets, while at least one b-tagged jet is required for the signal selection.

Main background sources in the analysis are either with three prompt leptons, or with two prompt leptons plus one non-prompt lepton. The former case includes the production of WZ, tt'Z, and $\mathrm{tZq}$ from SM. The later category covers the processes of Drell-Yan plus jets, $\mathrm{t} \overline{\mathrm{t}}$, or WW. The rate to find a non-prompt lepton is estimated by the data containing lepton candidates with inverted isolation requirement. Candidate events are categorized into two signal regions for single top and $t \bar{t}$ productions, and three control regions in order to estimate the background contributions. A simultaneous global fit is performed to resolve the background contamination in the signal regions. As shown in Fig. 5, the background suppression is extended by introducing boosted decision tree (BDT), which combines several kinematical variables and b-tagging information. Separate BDTs are trained for different lepton flavours, for single top or top pair categories, and for up or charm couplings. The FCNC process can be characterized using the effective theory approach,

$$
L_{F C N C}^{\mathrm{tZq}}=\sum_{q=u, c}\left[\frac{\sqrt{2}}{4} \frac{g}{\cos \theta_{W}} \frac{\kappa_{\mathrm{tZq}}}{\Lambda} \bar{t} \sigma^{\mu \nu}\left(f_{\mathrm{Zq}}^{L} P_{L}+f_{\mathrm{Zq}}^{R} P_{R}\right) q Z_{\mu v}\right]+h . c .,
$$

where $\kappa_{\mathrm{tZq}} / \Lambda$ represents the couplings at scale $\Lambda$. The upper limits are presented in either $\mathrm{t} \rightarrow \mathrm{cZ}$ and $\mathrm{t} \rightarrow \mathrm{uZ}$ branching fractions, or in $\kappa_{\mathrm{tZc}} / \Lambda$ and $\kappa_{\mathrm{tZu}} / \Lambda$, as shown in Fig. 6 . If only one of the two couplings is considered, the upper limits at the $95 \%$ confidence level on the $\mathrm{t} \rightarrow \mathrm{cZ}$ and $\mathrm{t} \rightarrow \mathrm{uZ}$ branching fractions are obtained to be $0.045 \%$ and $0.024 \%$, respectively.

\section{Search for Top FCNH decays with $\mathrm{H} \rightarrow \mathrm{b} \overline{\mathrm{b}}$}

The FCNC processes that correspond to $t$ and Higgs interaction (FCNH) can be described by 

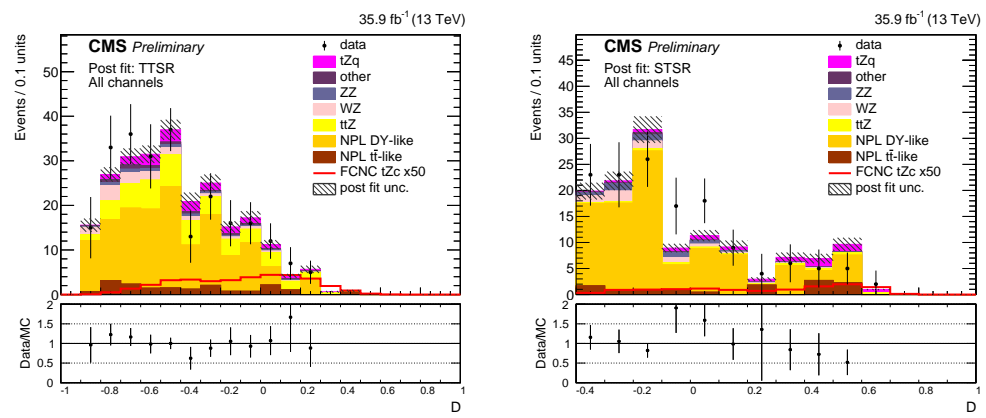

Figure 5: The post-fit BDT discriminating variable distributions for top quark pair tZc channel (left) and single top quark tZc (right). See Ref. [4] for detailed information.
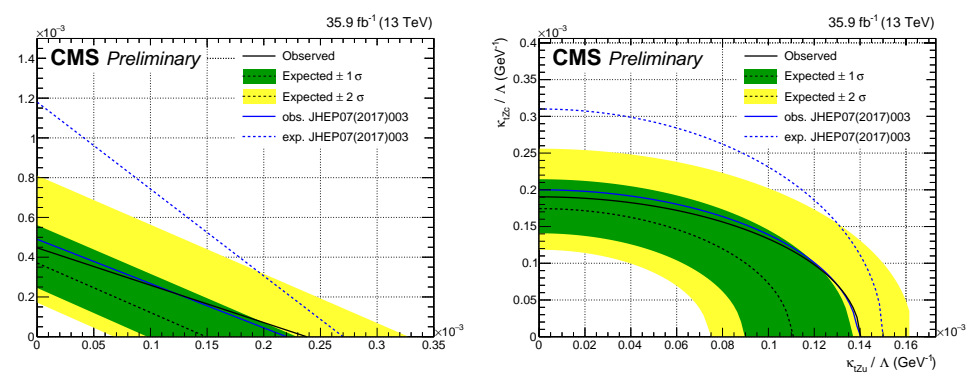

Figure 6: Exclusion regions at 95\% confidence level on the FCNC $t \rightarrow c Z$ and $t \rightarrow u Z$ branching fractions (left) and couplings (right). See Ref. [4] for detailed information.

the following effective Lagrangian:

$$
L=\sum_{q=u, c} \frac{g}{\sqrt{2}} \bar{t}_{\kappa_{\mathrm{HHq}}}\left(f_{H q}^{L} P_{L}+f_{H q}^{R} P_{R}\right) q H+\text { h.c. }
$$

where $\kappa_{\mathrm{qHt}}$ is the effective coupling. The top-Higgs FCNH interaction is studied in two channels as well: single top quark, or FCNH decays of top pair events. In this analysis [5] the $\mathrm{H} \rightarrow \mathrm{b} \overline{\mathrm{b}}$ decays are considered, and resulting a signature of one lepton plus three b-jets for the single top quark channel, and with one additional light-quark jet for the top pair events. The background, which is mostly dominant by the SM top pair events, contains one lepton, 2 b-jets, and 2 light-quark jets.

The event is reconstructed with full kinematical information under three event hypotheses, including semileptonic $t \bar{t}$, signal single top plus Higgs, signal $t \bar{t}$ with Higgs. For each jet permutation, a likelihood minimization is performed over momentum and energy resolution functions for the reconstructed leptons and jets. The assignment of $b$-jets is further improved by requiring an additional BDT trained on the candidates with correct or wrong permutations.

In each candidate event, three or four jets are required with two of them must be b-tagged. Events are categorized by either how many jets in total or how many jets are b-tagged. The discrimination of signal and background is further improved by a BDT trained separately for the two couplings $\mathrm{t} \rightarrow \mathrm{uH}$ and $\mathrm{t} \rightarrow \mathrm{cH}$, and for each event category, as shown in Fig. 7. Based on the standard $C L_{S}$ method, the limits at the $95 \%$ confidence level are obtained in $\mathrm{t} \rightarrow \mathrm{cH}$ and $\mathrm{t} \rightarrow \mathrm{uH}$ 
branching fractions, as shown in Fig. 8. If only one of the two couplings is considered, the upper limits on the $\mathrm{t} \rightarrow \mathrm{cH}$ and $\mathrm{t} \rightarrow \mathrm{uH}$ branching fractions are obtained to be both around $0.47 \%$. This is the first search for FCNH process in both single top plus Higgs, and top pair decaying into Higgs boson together, and is the best CMS limit on $\mathrm{t} \rightarrow \mathrm{uH}$ coupling.
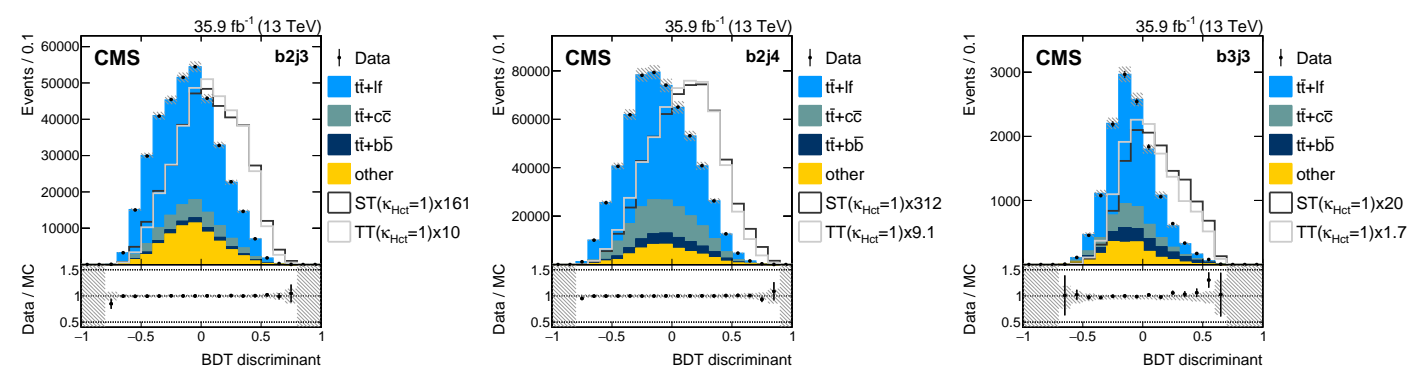

Figure 7: The post-fit BDT discriminant distributions for some different jet categories. The background processes are constrained to the SM expectations. See Ref. [5] for detailed information.

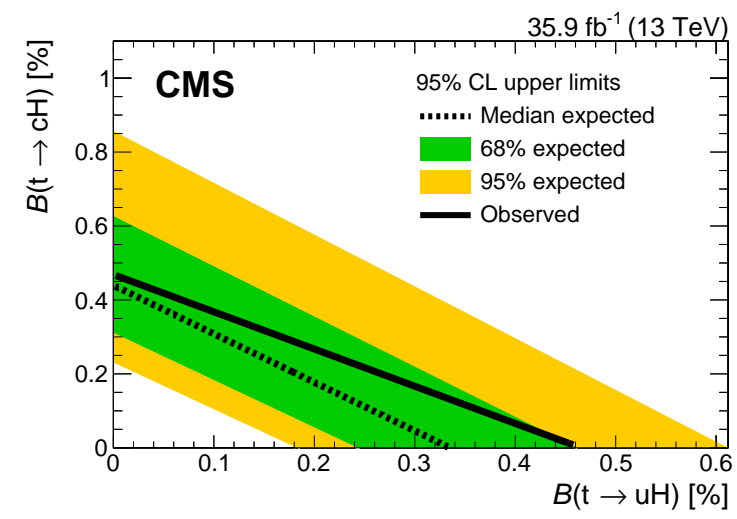

Figure 8: Exclusion regions at 95\% confidence level on the $\mathrm{FCNH} \mathrm{t} \rightarrow \mathrm{cH}$ and $\mathrm{t} \rightarrow \mathrm{uH}$ branching fractions. See Ref. [5] for detailed information.

\section{References}

[1] S. Chatrchyan et al. [CMS Collaboration], JINST 3, S08004 (2008). doi:10.1088/1748-0221/3/08/S08004

[2] A. M. Sirunyan et al. [CMS Collaboration], doi:10.3204/PUBDB-2018-02205 arXiv:1805.01428 [hep-ex].

[3] CMS Collaboration [CMS Collaboration], CMS-PAS-TOP-16-019, https://cds.cern.ch/record/2218019.

[4] CMS Collaboration [CMS Collaboration], CMS-PAS-TOP-17-017, https://cds.cern.ch/record/2292045.

[5] A. M. Sirunyan et al. [CMS Collaboration], doi:10.1007/JHEP06(2018)102 arXiv:1712.02399 [hep-ex]. 